CLINICAL STUDY

\title{
Growth differentiation factor 15 predicts future insulin resistance and impaired glucose control in obese nondiabetic individuals: results from the XENDOS trial
}

Tibor Kempf, Anja Guba-Quint, Jarl Torgerson ${ }^{1}$, Maria Chiara Magnone ${ }^{2}$, Carolina Haefliger ${ }^{2}$ Maria Bobadilla $^{2}$ and Kai C Wollert

Division of Molecular and Translational Cardiology, Department of Cardiology and Angiology, Hannover Medical School, CarlNeuberg-Strasse 1, 30625 Hannover, Germany, ${ }^{1}$ Department of Health Care, Region Västra Götaland, Göteborg, Sweden and ${ }^{2}$ F. Hoffmann-La Roche, Pharma Research and Early Development, Cardiovascular and Metabolic Disease Therapy Area, Basel, Switzerland

(Correspondence should be addressed to K C Wollert; Email: wollert.kai@mh-hannover.de)

\begin{abstract}
Objective: Growth differentiation factor-15 (GDF-15) is a stress-responsive cytokine that is increased in obesity and established type 2 diabetes. We assessed whether GDF-15 can predict future insulin resistance and impaired glucose control in obese nondiabetic individuals.

Design and methods: Plasma GDF-15 concentrations were measured with an automated electrochemiluminescent immunoassay at baseline and after 4 years in 496 obese nondiabetic individuals ( $52 \%$ men, median age 48 years, median body mass index (BMI) $37.6 \mathrm{~kg} / \mathrm{m}^{2}$ ) enrolled in the XENical in the prevention of Diabetes in Obese subjects (XENDOS) trial.

Results: The median GDF-15 concentration at baseline was $869 \mathrm{ng} / \mathrm{l}$ (interquartile range 723-1064 ng/l). GDF-15 was related to body weight, BMI, waist-to-hip ratio, and insulin resistance (homeostasis model assessment of insulin resistance (HOMA-IR)) (all $P<0.01$ ). Changes in GDF-15 from baseline to 4 years were related to changes in body weight, BMI, waist-to-hip ratio, and HOMA-IR (all $P<0.05$ ). Baseline GDF-15 was associated with the risk to have prediabetes or diabetes at 4 years by univariate analysis (odds ratio (OR) for 1 unit increase in ln GDF-15, 3.2; 95\% confidence interval (CI): 1.7-6.1; $P<0.001$ ), and after multivariate adjustment for age, gender, treatment allocation (orlistat vs placebo), BMI, waist-to-hip ratio, and glucose control at baseline (OR 2.2; 95\% CI: 1.1-4.7; $P=0.026)$. Similarly, baseline GDF-15 was independently associated with HOMA-IR at 4 years $(P=0.024)$.

Conclusions: This first longitudinal study of GDF-15 in a large cohort of obese individuals indicates that GDF-15 is related to abdominal obesity and insulin resistance and independently associated with future insulin resistance and abnormal glucose control.
\end{abstract}

European Journal of Endocrinology 167 671-678

\section{Introduction}

Obesity prevalence has increased in the past 30 years in most countries and regions of the world (1). The obesity epidemic is driven mainly by the increased availability and promotion of cheap, energy-dense food in combination with low physical activity levels (2). The global rise of obesity has serious health effects. Obesity is a leading cause of type 2 diabetes and its main complication, cardiovascular disease (3).

Type 2 diabetes is at one end of a continuous glucose control spectrum with normal glucose tolerance at the other. In between, there exists a condition called prediabetes, defined as impaired fasting glucose (IFG) or impaired glucose tolerance (IGT) (4). Like diabetes, prediabetes is associated with obesity (especially abdominal obesity), insulin resistance, dyslipidemia with high triglycerides and/or low HDL cholesterol, and hypertension (4). Recent data suggest that $12.9 \%$ of the adult US population have diabetes and another $29.5 \%$ have prediabetes (5). Individuals with prediabetes have a high risk of developing diabetes (4), and a sizable increase in the risk of cardiovascular disease (6). Given these factors, individuals with prediabetes will form a significant proportion of the health care burden associated with obesity in the future. Because of the inter-individual variability in the risk of developing prediabetes or diabetes, identification of obese individuals at higher risk is important to target and intensify preventive measures.

Growth differentiation factor-15 (GDF-15), initially described as macrophage-inhibitory cytokine 1 (7), is a member of the transforming growth factor- $\beta$ cytokine 
superfamily that is produced in response to oxidative stress and inflammation by multiple cell types, including macrophages, adipocytes, and cardiovascular cells $(7,8,9,10)$. Increased circulating concentrations of GDF-15 are associated with increased risks of fatal and nonfatal cardiovascular events in community-dwelling individuals $(11,12)$, and patients with cardiovascular disease $(13,14,15,16)$. In these studies, individuals with established type 2 diabetes had higher circulating concentrations of GDF-15, also after adjustment for confounding factors including body weight. Two smaller, cross-sectional studies found that 'healthy' obese individuals also have increased circulating levels of GDF-15 which were found to be associated with measures of impaired glucose control $(9,17)$.

These previous reports prompted us to explore in a longitudinal study of obese nondiabetic individuals enrolled in the XENDOS trial i) whether GDF-15 plasma levels rise already in a prediabetic, insulin-resistant state and ii) whether GDF-15 can predict future insulin resistance and impaired glucose control.

\section{Materials and methods}

\section{Patients and study design}

The present investigation is a substudy from the XENDOS trial, a 4-year, double-blind, randomized, and placebo-controlled study carried out at 22 Swedish medical centers between 1997 and 2002. The protocol and main results of the trial have been published (18). Eligible individuals were 30-60 years of age, with a BMI $\geq 30 \mathrm{~kg} / \mathrm{m}^{2}$. Participants were required to have a nondiabetic glucose tolerance as assessed by a $75 \mathrm{~g}$ oral glucose tolerance test (OGTT) performed at baseline using venous whole blood and the 1994 WHO criteria (fasting whole blood glucose $<6.7 \mathrm{mmol} / \mathrm{l}$ and 2 -h whole blood glucose $<10.0 \mathrm{mmol} / \mathrm{l})$. Patients with cardiovascular or gastrointestinal disease were excluded (18). During the study, all participants were prescribed a reduced-calorie diet $(\sim 800 \mathrm{kcal}$ deficit per day) containing $30 \%$ of calories from fat and not more than $300 \mathrm{mg}$ cholesterol per day. The prescribed energy intake was readjusted every 6 months to account for any weight loss during the preceding months. Participants received dietary counseling on a regular basis and were randomized in a one-to-one ratio to placebo or orlistat $120 \mathrm{mg}$ with breakfast, lunch, and dinner. In the overall trial, $52 \%$ of the orlistat patients compared with $34 \%$ of the placebo patients completed treatment. Mean weight loss after 4 years was significantly greater with orlistat compared with placebo (5.8 vs $3.0 \mathrm{~kg}$ ) (18). Estimated glomerular filtration rate (eGFR) was calculated by the CKD-EPI equation (19). Metabolic syndrome was defined according to a recent Joint Scientific Statement (20). The present biomarker substudy includes 496 individuals from both randomization groups. The study protocol was approved by all relevant ethics review committees in Sweden. All study participants provided written informed consent.

\section{Assessment of glucose control}

Fasting plasma insulin and venous whole blood glucose, as well as 2-h plasma insulin and venous whole blood glucose concentrations from a $75 \mathrm{~g}$ OGTT were available at baseline and at 4 years. A constant factor of 1.11 was used to convert glucose concentration in whole blood to the equivalent concentration in the pertinent plasma (21). Diagnosis and classification of prediabetes and diabetes were performed according to the recent position statement of the American Diabetes Association (4). Diabetes was defined as a fasting plasma glucose concentration $\geq 7.0 \mathrm{mmol} / \mathrm{l}$ or a 2 -h OGTT plasma glucose concentration $\geq 11.1 \mathrm{mmol} / \mathrm{l}$. IFG was defined as a fasting plasma glucose $\geq 5.6$ and $\leq 6.9 \mathrm{mmol} / \mathrm{l}$, and a 2-h OGTT plasma glucose $<7.8 \mathrm{mmol} / \mathrm{l}$. IGT was defined as a fasting plasma glucose $<7.0 \mathrm{mmol} / \mathrm{l}$ and a 2-h OGTT plasma glucose $\geq 7.8$ and $<11.1 \mathrm{mmol} / \mathrm{l}$. Homeostasis model assessment of insulin resistance (HOMA-IR) was calculated as fasting plasma insulin (in $\mathrm{mU} / \mathrm{l}) \times$ fasting plasma glucose (in mmol/l)/22.5 (22).

\section{GDF-15 measurements}

At baseline and at 4 years, GDF-15 was measured in morning EDTA plasma samples collected after an overnight fast. Participants were supine in position for 5-10 min prior to phlebotomy. Blood was immediately centrifuged, and plasma was stored at $-70{ }^{\circ} \mathrm{C}$ until assayed. GDF-15 was measured with a precommercial, automated electrochemiluminescent immunoassay on a cobas e 411 analyzer (Roche Diagnostics). The assay has a limit of detection below $10 \mathrm{ng} / \mathrm{l}$, a linear measuring range up to $20000 \mathrm{ng} / \mathrm{l}$, and an inter-assay imprecision of 2.3 and $1.8 \%$ at GDF-15 concentrations of 1100 and $17200 \mathrm{ng} / \mathrm{l}$, respectively (Roche Diagnostics, data on file). GDF-15 values obtained with the electrochemiluminescent assay correlate closely with the values measured with our previously described (23) IRMA $(r=0.980$, slope 1.049 , intercept $-136 \mathrm{ng} / \mathrm{l}, n=45$ samples with GDF-15 concentrations ranging from 567 to $13334 \mathrm{ng} / \mathrm{l})$.

\section{Statistical analysis}

Data are presented as numbers (\%) or median (interquartile range). The Kolmogorov-Smirnov test was used to test for a normal distribution of continuous variables. GDF-15 and HOMA-IR were not normally distributed and were transformed to their natural logarithm for all regression analyses. Continuous 
Table 1 Study population. Data are shown as numbers (\%) or median (interquartile range).

\begin{tabular}{|c|c|c|c|}
\hline & Baseline & 4 Years & $P$ \\
\hline$n$ & 496 & 496 & \\
\hline Age (years) & $48(40-52)$ & $52(44-56)$ & \\
\hline Male gender & $258(52)$ & $258(52)$ & \\
\hline Body weight $(\mathrm{kg})$ & $112.4(101.3-124.2)$ & $108.1(97.3-121.1)$ & $<0.001$ \\
\hline $\mathrm{BMI}\left(\mathrm{kg} / \mathrm{m}^{2}\right)$ & $37.6(35.3-40.7)$ & $36.4(33.5-39.5)$ & $<0.001$ \\
\hline Waist-to-hip ratio & $1.00(0.94-1.04)$ & $0.98(0.93-1.03)$ & $<0.001$ \\
\hline Fasting glucose $(\mathrm{mmol} / \mathrm{l})$ & $5.3(4.9-5.8)$ & $5.4(5.1-6.0)$ & $<0.001$ \\
\hline Fasting insulin $(\mathrm{pmol} / \mathrm{l})$ & $84(60-120)$ & $62(37-94)$ & $<0.001$ \\
\hline HOMA-IR & $2.8(1.9-4.2)$ & $2.1(1.2-3.3)$ & $<0.001$ \\
\hline 2-h OGTT glucose $(\mathrm{mmol} / \mathrm{l})$ & $7.1(5.8-8.5)$ & $6.7(5.3-8.2)$ & 0.022 \\
\hline 2-h OGTT insulin (pmol/l) & $384(222-635)$ & $283(157-457)$ & $<0.001$ \\
\hline LDL cholesterol $(\mathrm{mmol} / \mathrm{l})$ & $3.7(3.2-4.3)$ & $3.3(2.8-3.9)$ & $<0.001$ \\
\hline HDL cholesterol ( $\mathrm{mmol} / \mathrm{l})$ & $1.1(1-1.3)$ & $1.2(1.0-1.4)$ & $<0.001$ \\
\hline Triglycerides $(\mathrm{mmol} / \mathrm{l})$ & $1.8(1.4-2.4)$ & $1.8(1.3-2.4)$ & 0.43 \\
\hline $\mathrm{SBP}(\mathrm{mmHg})$ & $135(123-145)$ & $130(120-140)$ & $<0.001$ \\
\hline eGFR $\left(\mathrm{ml} / \mathrm{min}\right.$ per $\left.1.73 \mathrm{~m}^{2}\right)$ & $85(77-94)$ & $84(74-92)$ & 0.040 \\
\hline GDF-15 (ng/l) & $869(723-1064)$ & $932(756-1141)$ & 0.003 \\
\hline
\end{tabular}

HOMA-IR, homeostasis model assessment of insulin resistance; OGTT, oral glucose tolerance test; $\mathrm{SBP}$, systolic blood pressure; eGFR, estimated glomerular filtration rate.

variables were compared by the Mann-Whitney $U$ test. Comparisons between strata of patients were performed by one-way ANOVA with Fisher's post hoc test. Proportions were compared by the $\chi^{2}$ test. Simple and multivariate linear regression analyses were used to evaluate the association of clinical and biochemical parameters with ln GDF-15 or In HOMA-IR as dependent variables. Simple and multivariate logistic regression analyses were used to identify predictors of an abnormal glucose control (prediabetes or diabetes) at 4 years. All $P$ values are reported two-sided. $P$ values $<0.05$ were considered to indicate statistical significance. No adjustments for multiplicity were made and the results are to be considered exploratory. Analyses were performed using the StatView 5 and SPSS 19 statistical programs.

\section{Results}

\section{Study population}

The study population included 496 individuals with a balanced gender distribution ( $52 \%$ men) and a median age of 48 years (interquartile range $40-52$ years). Study participants had a median body weight of $112.4 \mathrm{~kg}$, a median BMI of $37.6 \mathrm{~kg} / \mathrm{m}^{2}$, and a median waist-to-hip ratio of 1.0 (Table 1 ). After 4 years, study participants had lost on average (mean) $3.9 \mathrm{~kg}$ which was associated with improved HOMA-IR, an improved lipid profile (increased HDL and reduced LDL cholesterol levels), and a reduction in systolic blood pressure (Table 1).

At baseline, 238 individuals (48\%) had a normal glucose tolerance and $258(52 \%)$ had prediabetes. After 4 years, 235 individuals (47\%) had a normal glucose tolerance, 217 (44\%) had prediabetes, and 44 (9\%) had diabetes. Among those with a normal glucose tolerance at baseline, $35 \%$ (83 out of 238) had developed prediabetes at 4 years and 4\% (9/238) had developed diabetes; among individuals with prediabetes at baseline, $34 \%(89 / 258)$ had a normal glucose tolerance at 4 years and 14\% (35/258) had progressed to diabetes. Overall, 216 individuals (44\%) had changed their glucose control status from baseline to 4 years.

\section{GDF-15 in relation to clinical parameters and glucose control at baseline}

The median GDF-15 concentration at baseline was $869 \mathrm{ng} / \mathrm{l}$ (interquartile range $723-1064 \mathrm{ng} / \mathrm{l}$; range 384-2654 ng/l). GDF-15 was related to age, male gender, anthropometric measures of obesity (body weight, BMI, waist-to-hip ratio), impaired glucose control (fasting insulin, HOMA-IR, 2-h OGTT glucose),

Table 2 Baseline variables associated with GDF-15. All baseline variables shown in Table 1 were tested for their univariate and multivariate (independent) associations with In GDF-15 at baseline. Body weight, fasting glucose and insulin, and 2-h OGTT glucose and insulin were not included in the multivariate analysis, given that BMI and HOMA-IR were considered. Statistically significant associations are shown.

\begin{tabular}{lrrrrr}
\hline & \multicolumn{2}{c}{ Univariate } & & \multicolumn{2}{c}{ Multivariate } \\
\cline { 2 - 3 } \cline { 5 - 6 } & \multicolumn{1}{c}{$r$} & \multicolumn{1}{c}{$P$} & & $\begin{array}{l}\text { Standar- } \\
\text { dized } \beta\end{array}$ & $P$ \\
\hline Age & 0.38 & $<0.001$ & & 0.31 & $<0.001$ \\
Male gender & 0.15 & 0.001 & & \\
Body weight & 0.14 & 0.003 & & \\
BMl & 0.15 & 0.001 & & 0.14 & 0.002 \\
Waist-to-hip ratio & 0.12 & 0.006 & & \\
Fasting insulin & 0.14 & 0.002 & & \\
HOMA-IR & 0.15 & $<0.001$ & & \\
2-h OGTT glucose & 0.10 & 0.024 & & \\
Triglycerides & 0.11 & 0.016 & & \\
SBP & 0.24 & $<0.001$ & & \\
eGFR & -0.22 & $<0.001$ & & -0.16 & 0.001 \\
\hline
\end{tabular}


A

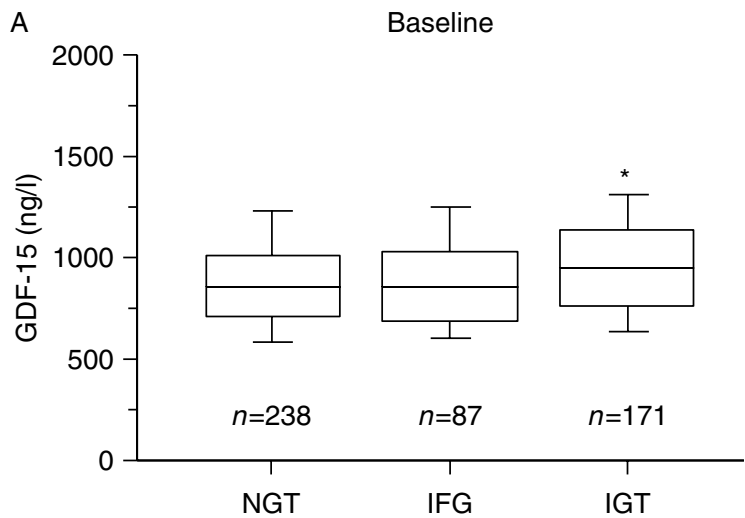

B

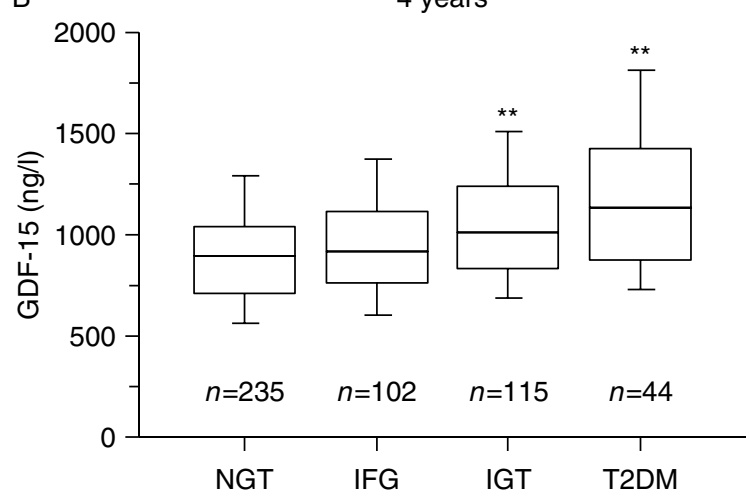

Figure $1 \mathrm{GDF}-15$ concentrations in relation to glucose control at baseline (A) and 4 years (B). GDF-15 plasma concentrations are presented as box (median with interquartile range) and whisker (10th and 90th percentiles) plots. NGT, normal glucose tolerance; IFG, impaired fasting glucose; IGT, impaired glucose tolerance; T2DM, type 2 diabetes mellitus. ${ }^{\star} P<0.01,{ }^{\star \star} P<0.001$ vs NGT (ANOVA with Fisher's post hoc test).

triglycerides, systolic blood pressure, and lower eGFR (Table 2). Upon multivariate adjustment (Table 2), GDF-15 remained independently associated with age $(P<0.001)$, BMI $(P=0.002)$, and lower eGFR $(P=0.001)$. Individuals with prediabetes had somewhat higher GDF-15 concentrations compared with those with a normal glucose tolerance (median (interquartile range) 893 (746-1087) vs 854 (712-1007) ng/l; $P=0.046)$. Among individuals with prediabetes, GDF15 levels were elevated in IGT but not in IFG (Fig. 1A).

\section{Change of GDF-15 concentration over time}

At 4 years, the median GDF-15 concentration had increased slightly to 932 (interquartile range 756-1141) ng/l, representing a median increase from baseline of $42 \mathrm{ng} / \mathrm{l}$ (interquartile range -65 to $166 \mathrm{ng} / \mathrm{l}$; range -1350 to $2315 \mathrm{ng} / \mathrm{l} ; \quad P<0.001$ ). The inter-individual variability of the changes in GDF-15 from baseline to 4 years is illustrated in Fig. 2.

Sixty-eight percent of the study participants fulfilled the criteria for metabolic syndrome at baseline, $66 \%$ at
4 years. At 4 years, GDF-15 levels were higher in patients with metabolic syndrome compared with those without (959 (794-1177) vs 875 (708-1048) ng/l, $P<0.001)$. No significant difference was observed at baseline $(P=0.11)$.

GDF-15 levels at baseline were comparable in patients randomized to placebo or orlistat $(P=0.91)$. Changes over 4 years were not significantly different between both groups (median change (interquartile range), placebo 59 ( -56 to 169$)$ vs orlistat $25(-73$ to 156); $P=0.12$ ).

Also at 4 years, GDF-15 concentrations were higher in individuals with prediabetes than in those with a normal glucose tolerance (941 (interquartile range 806-1189) vs 896 (709-1047) ng/l; $P=0.001)$; this resulted again from increased GDF-15 levels in IGT but not in IFG (Fig. 1B). Even higher concentrations were measured in patients with type 2 diabetes at 4 years (1136 (876-1428) ng/l; $P<0.001$ vs normal glucose tolerance) (Fig. 1B).

The same baseline variables (except for body weight, 2-h OGTT glucose, and eGFR) that were associated with GDF-15 at study entry were associated with changes in GDF-15 over time (Table 3). Moreover, changes in body weight, BMI, and waist-to-hip ratio; fasting glucose, fasting insulin, 2-h OGTT glucose, and 2-h OGTT insulin; HOMA-IR; triglyceride levels; and eGFR (inverse relation) were associated with changes in GDF-15 over time (Table 3). Upon multivariate adjustment, age $(P<0.001)$, changes in waist-to-hip ratio $(P=0.025)$ and triglycerides $(P=0.023)$, HOMA-IR $(P=0.006)$, and eGFR $(P=0.020$, inverse relation) remained independently associated with changes in GDF-15 (Table 3).

\section{GDF-15 at baseline in relation to glucose control at 4 years}

The relationship between baseline GDF-15 and glucose control at 4 years is illustrated in Fig. 3. The risks of an abnormal glucose control (prediabetes or diabetes) at

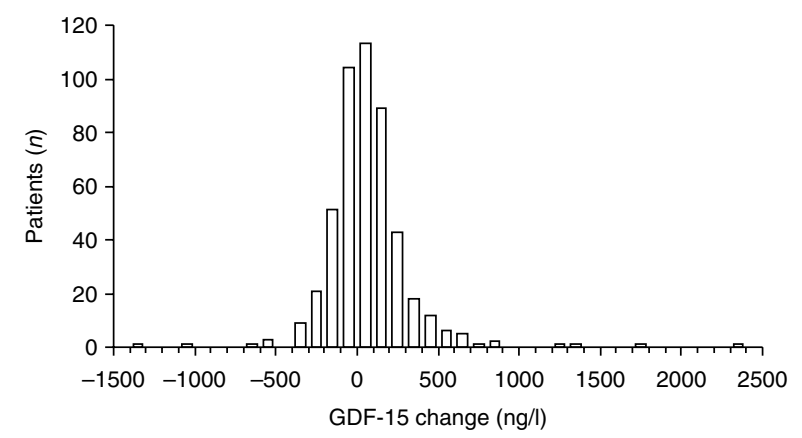

Figure 2 Changes in GDF-15 concentrations from baseline to 4 years. Distribution of the change of GDF-15 plasma concentrations over time. 
Table 3 Baseline variables and their changes over 4 years associated with changes of GDF-15 from baseline to 4 years. The baseline variables shown in Table 1 , their changes $(\Delta)$ from baseline to 4 years (where applicable), and treatment allocation were tested for their univariate and multivariate (independent) associations with change in GDF-15 from baseline to 4 years. The multivariate model was adjusted for baseline In GDF-15. Body weight, fasting glucose and insulin, and 2-h OGTT glucose and insulin (and the changes thereof) were not included into the multivariate analysis, given that BMI and HOMA-IR (and their changes) were considered. Statistically significant associations are shown.

\begin{tabular}{lrrrrr}
\hline & \multicolumn{2}{c}{$\begin{array}{c}\text { Univariate linear } \\
\text { regression }\end{array}$} & & \multicolumn{2}{c}{ Multivariate } \\
\cline { 2 - 3 } \cline { 5 - 6 } & \multicolumn{1}{c}{$r$} & $P$ & $\begin{array}{c}\text { Standard- } \\
\text { ized } \beta\end{array}$ & $P$ \\
\hline Age & 0.38 & $<0.001$ & & 0.29 & $<0.001$ \\
Male gender & 0.15 & 0.001 & & \\
$\Delta$ Body weight & 0.10 & 0.025 & & \\
BMl & 0.15 & 0.001 & & \\
$\Delta$ BMI & 0.10 & 0.034 & & \\
Waist-to-hip ratio & 0.12 & 0.006 & & \\
$\Delta$ Waist-to-hip ratio & 0.12 & 0.009 & 0.11 & 0.025 \\
$\Delta$ Fasting glucose & 0.17 & $<0.001$ & & \\
Fasting insulin & 0.13 & 0.004 & & \\
$\Delta$ Fasting insulin & 0.18 & $<0.001$ & & \\
HOMA-IR & 0.10 & 0.033 & & \\
$\Delta$ HOMA-IR & 0.19 & $<0.001$ & 0.16 & 0.006 \\
$\Delta$ 2-h OGTT glucose & 0.19 & $<0.001$ & & \\
$\Delta$ 2-h OGTT insulin & 0.17 & $<0.001$ & & \\
Triglycerides & 0.11 & 0.016 & & \\
$\Delta$ Triglycerides & 0.17 & $<0.001$ & 0.12 & 0.023 \\
SBP & 0.24 & $<0.001$ & & \\
eGFR & -0.05 & 0.31 & & \\
$\Delta$ eGFR & -0.12 & 0.006 & -0.10 & 0.020 \\
\hline
\end{tabular}

4 years increased with increasing tertiles of baseline GDF-15 $(P=0.021)$.

The risk to have an abnormal glucose control at 4 years increased with increasing GDF-15 concentrations at baseline (odds ratio (OR) for 1 unit increase in $\mathrm{ln}$ GDF-15 3.2; 95\% confidence interval: 1.7-6.1; $P<0.001$ ). In a multivariable logistic regression analysis that adjusted for age, gender, treatment allocation (orlistat vs placebo), baseline BMI and waist-to-hip ratio, and stratum at baseline (normal glucose tolerance vs prediabetes), baseline GDF-15 remained significantly associated with the risk to have an abnormal glucose control at 4 years (Table 4). Besides GDF-15, only prediabetes at baseline emerged as an independent predictor of an abnormal glucose control at 4 years (Table 4).

To complement these analyses relating baseline GDF-15 to a dichotomous outcome at 4 years (normal vs abnormal glucose control), we assessed the relationship of baseline GDF-15 with HOMA-IR at 4 years. Increasing GDF-15 concentrations at baseline was significantly associated with HOMA-IR at 4 years (Pearson's $r=0.22 ; P<0.001)$. After multivariate adjustment for age, gender, treatment allocation, baseline BMI and waist-to-hip ratio, and baseline HOMA-IR,
GDF-15 at baseline remained significantly associated with HOMA-IR at 4 years (standardized $\beta=0.10$; $P=0.024)$. Other variables independently associated with HOMA-IR at 4 years included baseline HOMA-IR (standardized $\beta=0.37 ; P<0.001$ ), baseline BMI (standardized $\beta=0.20 ; P<0.001$ ), and allocation to the placebo group (standardized $\beta=0.12 ; P=0.003$ ).

\section{Discussion}

The present longitudinal study in a large cohort of obese individuals identifies GDF-15 as a marker of abdominal obesity and insulin resistance and shows that GDF-15 levels at baseline provide independent information on insulin resistance and glucose control 4 years later.

GDF-15 levels in this study were higher than the levels previously measured in apparently healthy nonobese, 40-59 year-old Swedish individuals $(n=110$; median age 56 years; median GDF-15 $667 \mathrm{ng} / \mathrm{l}$; interquartile range 487-869 ng/l; $P<0.001$ ) (23), but lower than the levels in patients with established cardiovascular disease $(13,14,15,16)$. GDF-15 levels were higher in obese individuals with prediabetes compared with those with a normal glucose tolerance. GDF-15 was not related to fasting glucose, and individuals with IFG did not present with higher GDF-15 levels compared with those having a normal glucose tolerance. Instead, GDF-15 was high in individuals with IGT, which is reflecting postprandial peripheral insulin resistance (24), and appears to be associated with higher cardiovascular risk as compared with IFG (25). In addition, GDF-15 was associated with fasting insulin and insulin resistance (HOMA-IR) which are reflecting whole body insulin resistance (22).

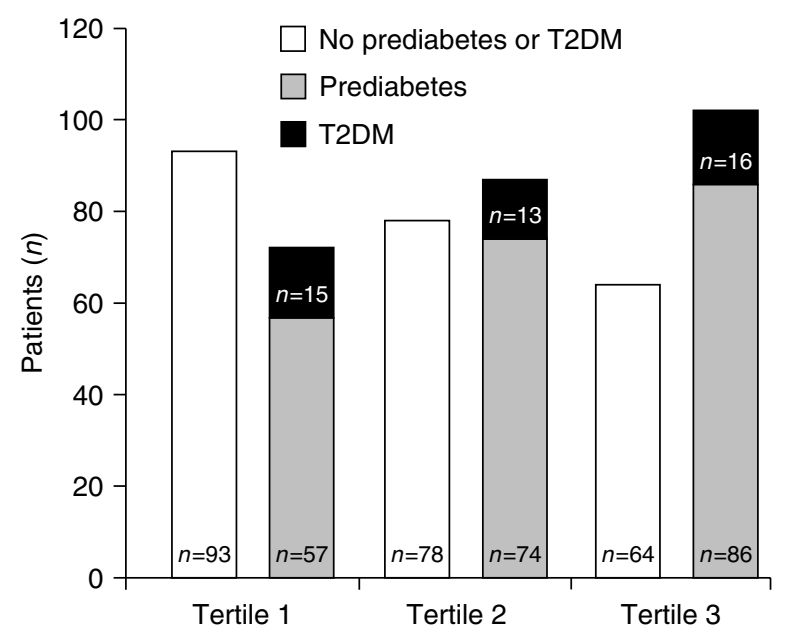

Figure 3 Glucose control at 4 years in relation to GDF-15 concentrations at baseline. Number of patients with normal glucose tolerance, prediabetes, or type 2 diabetes (T2DM) at 4 years in relation to tertiles of GDF-15 plasma concentrations at baseline (tertile boundaries, 772 , and $979 \mathrm{ng} / \mathrm{l}$ ). $P=0.021$ ( $\chi^{2}$ test). 
Table 4 Risk of abnormal glucose control (prediabetes or diabetes) at 4 years in relation to baseline variables and treatment allocation. All variables were entered into the multivariable logistic regression analysis; statistically significant associations are shown.

\begin{tabular}{|c|c|c|c|c|}
\hline & \multicolumn{2}{|c|}{ Univariate } & \multicolumn{2}{|c|}{ Multivariate } \\
\hline & OR $(95 \% \mathrm{Cl})$ & $P$ & OR $(95 \% \mathrm{Cl})$ & $P$ \\
\hline Age & $1.0(1.0-1.1)$ & 0.003 & & \\
\hline Male gender & $1.3(0.9-1.8)$ & 0.19 & & \\
\hline BMI & $1.0(1.0-1.1)$ & 0.46 & & \\
\hline $\begin{array}{l}\text { Waist-to-hip } \\
\text { ratio }\end{array}$ & $4.8(0.4-63)$ & 0.23 & & \\
\hline $\begin{array}{c}\text { Prediabetes at } \\
\text { baseline }\end{array}$ & $3.0(2.1-4.4)$ & $<0.001$ & $2.8(1.9-4.1)$ & $<0.001$ \\
\hline $\begin{array}{l}\text { Allocation to } \\
\text { orlistat }\end{array}$ & $0.8(0.6-1.1)$ & 0.19 & & \\
\hline In GDF-15 & $3.2(1.7-6.1)$ & $<0.001$ & $2.2(1.1-4.7)$ & 0.026 \\
\hline
\end{tabular}

$\mathrm{Cl}$, confidence interval.

In keeping with this observation, worsening insulin resistance over time in individuals who developed type 2 diabetes at 4 years was associated with the highest GDF-15 concentrations.

We observed a considerable inter-individual variability in the changes in GDF-15 over time. Age emerged as the strongest predictor of baseline GDF-15 and its changes. In addition, changes in GDF-15 were related to changes in abdominal obesity and insulin resistance. A previous study in 28 morbidly obese individuals undergoing gastric bypass surgery found GDF-1 5 levels to increase 1 year after the surgery, and noted that patients with larger reductions in body weight or greater improvements in insulin resistance had smaller increases in GDF-15 (17). Observations in communitydwelling individuals and patients with cardiovascular disease confirm the strong impact of age on GDF-15 $(11,12,13,14,15,26)$. However, age is not the only predictor of baseline GDF-15 and its changes; consistent with others (17), we identified obesity and insulin resistance as important modulators of baseline GDF-15 and its changes over time.

Our observations extend previous, smaller crosssectional studies that found GDF-15 to be associated with body weight, insulin resistance, and type 2 diabetes in obese individuals $(9,17)$. Associations of GDF-15 to obesity and diabetes have also been observed in community-dwelling individuals $(11,12,26)$. Moreover, diabetes is a main determinant of GDF-15 levels in patients with established cardiovascular disease $(13,14,15)$. Our study in obese individuals with no overt cardiovascular disease emphasizes that GDF-15 levels may start to increase already in a prediabetic, insulin resistant state before the occurrence of cardiovascular complications. The associations of GDF-15 to insulin resistance and prediabetes may help explain how GDF-15, as a biomarker, enhances cardiovascular risk assessment beyond age and other traditional cardiovascular risk factors in community-dwelling individuals and patients with cardiovascular disease $(11,12,13,14,15,16)$.

One of the most notable findings in this study was that GDF-15 levels at baseline were associated with the risk to have an abnormal glucose control at 4 years, an association that was independent of age, gender, treatment allocation, BMI and waist-to-hip ratio, and glucose control at baseline. Similarly, we observed an independent association of baseline GDF-15 to insulin resistance at 4 years. A nested case-control study in British civil servants, most of whom were not obese, found that GDF-15 levels at baseline were higher in individuals who subsequently developed type 2 diabetes compared with those who remained free of diabetes; this association, however, was no longer statistically significant after adjusting for age, gender, and waist circumference (27). In contrast to that report, participants in our study were obese, half of them already had prediabetes at baseline, and we followed them for any abnormal glucose control (prediabetes or diabetes) or insulin resistance. Thus, by design, our study may have been more sensitive to detect an independent association of GDF-15 with worsening glucose control.

While the cellular sources of GDF-15 in obesity and (pre)diabetes remain to be established, it is known that GDF-15 is expressed in human adipose tissue and is secreted from human adipocytes $(8,9)$. Expression of GDF-15 is controlled on a transcriptional level by p53 (10), a transcription factor that is thought to provide a critical link between obesity and insulin resistance (28). Indeed, obesity promotes p53 activation in adipose tissue thus leading to increased production of proinflammatory cytokines, insulin resistance, and diabetes (29). GDF-15 may be a marker of this disease pathway in adipose tissue.

Both insulin resistance and GDF-15 are associated with low-grade inflammation, which is reflected in multiple tissues, including adipose tissue and the vascular endothelium $(26,30,31)$. In keeping with this fact, insulin resistance and GDF-15 are both associated with endothelial dysfunction (26, 32). Hence, the association between GDF-15 and insulin resistance may also reflect an overall state of metabolic derangement, inflammation, and vascular pathologies that is associated with increased cardiovascular risk.

More studies are needed to conclude if GDF-15 is a marker, a causal mediator, or a compensatory mechanism in humans with obesity and insulin resistance, and to determine how this may relate to the increased cardiovascular risk observed in these individuals. Notably, transgenic over-expression of GDF-15 in mice leads to decreased body weight and fat mass and improved glucose tolerance under high fat-fed conditions, suggesting that GDF-15 may serve a compensatory role (33). Studies on GDF-15 deficient mice are required to assess the function of endogenous GDF-15 in this setting. 
Our study has a number of strengths and limitations that merit consideration. The size of the study population, the longitudinal design, and the careful and standardized clinical and biochemical follow-up within a clinical trial represent the strengths of our study. Participants in the trial were prescribed a reduced-calorie diet, they received dietary counseling and, by design, half of them were prescribed orlistat. As a potential limitation, some of our observations may therefore not apply to obese individuals not enrolled in similar weight reduction programs. Many of the baseline and change variables examined for their association with baseline GDF-15 and changes in GDF-15 over time are correlated, thus raising an issue of multicollinearity. Individual predictors identified (or eliminated) in the multiple regression models therefore need to be interpreted with caution. The number of obese individuals progressing to overt type 2 diabetes during 4 years of follow-up was small, and we were unable to explore if baseline GDF-15 predicts the development of diabetes. Finally, plasma samples were stored at $-70{ }^{\circ} \mathrm{C}$ for up to 15 years (4 years less in the follow-up samples). While GDF-15 is thought to have favorable preanalytical characteristics (23), the stability of the analyte during prolonged storage is difficult to assess directly. However, our conclusion that worsening cardiometabolic status is responsible for the observed increases in GDF-15 over time is consistent with previous studies on obese individuals and patients with heart failure $(14,17)$.

In conclusion, this study identifies GDF-15 as an independent predictor of insulin resistance and abnormal glucose control in obese individuals. GDF-15 may provide a link between obesity, diabetes, and cardiovascular risk, and our findings should therefore encourage further research into the pathobiology and prognostic implications of this cytokine in the setting of obesity and insulin resistance.

\section{Declaration of interest}

Drs T Kempf and K C Wollert are named as co-inventors on a patent for the use of GDF-15 for cardiovascular applications, and have a contract with Roche Diagnostics for the development of a GDF-15 assay. Drs M C Magnone, C Haefliger, and M Bobadilla are employees of F Hoffmann-La Roche. Mrs A Guba-Quint and Dr J Torgerson declare no potential conflict of interest.

\section{Funding}

Dr K C Wollert was supported by the German Ministry of Education and Research (BMBF, BioChancePlus). GDF-15 immunoassays were provided by Roche Diagnostics. The funders had no role in the design and conduct of the study or the collection and analysis of the data.

\section{References}

1 Finucane MM, Stevens GA, Cowan MJ, Danaei G, Lin JK, Paciorek CJ, Singh GM, Gutierrez HR, Lu Y, Bahalim AN,
Farzadfar F, Riley LM \& Ezzati M. National, regional, and global trends in body-mass index since 1980: systematic analysis of health examination surveys and epidemiological studies with 960 country-years and 9.1 million participants. Lancet 2011377 557-567. (doi:10.1016/S0140-6736(10)62037-5)

2 Swinburn BA, Sacks G, Hall KD, McPherson K, Finegood DT, Moodie ML \& Gortmaker SL. The global obesity pandemic: shaped by global drivers and local environments. Lancet 2011 378 804-814. (doi:10.1016/S0140-6736(11)60813-1)

3 Mazzone T, Chait A \& Plutzky J. Cardiovascular disease risk in type 2 diabetes mellitus: insights from mechanistic studies. Lancet 2008 371 1800-1809. (doi:10.1016/S0140-6736(08)60768-0)

4 American Diabetes Association. Diagnosis and classification of diabetes mellitus. Diabetes Care 201235 (Suppl 1) S64-S71.

5 Cowie CC, Rust KF, Ford ES, Eberhardt MS, Byrd-Holt DD, Li C, Williams DE, Gregg EW, Bainbridge KE, Saydah SH \& Geiss LS. Full accounting of diabetes and pre-diabetes in the U.S. population in 1988-1994 and 2005-2006. Diabetes Care 200932 287-294. (doi:10.2337/dc08-1296)

6 Ford ES, Zhao G \& Li C. Pre-diabetes and the risk for cardiovascular disease: a systematic review of the evidence. Journal of the American College of Cardiology 201055 1310-1317. (doi:10.1016/j.jacc. 2009.10.060)

7 Bootcov MR, Bauskin AR, Valenzuela SM, Moore AG, Bansal M, He XY, Zhang HP, Donnellan M, Mahler S, Pryor K, Walsh BJ, Nicholson RC, Fairlie WD, Por SB, Robbins JM \& Breit SN. MIC-1, a novel macrophage inhibitory cytokine, is a divergent member of the TGF- $\beta$ superfamily. PNAS $1997 \mathbf{9 4} 11514-11519$. (doi:10.1073/pnas.94.21.11514)

8 Ding Q, Mracek T, Gonzalez-Muniesa P, Kos K, Wilding J, Trayhurn P \& Bing C. Identification of macrophage inhibitory cytokine- 1 in adipose tissue and its secretion as an adipokine by human adipocytes. Endocrinology 2009150 1688-1696. (doi:10.1210/en.2008-0952)

9 Dostalova I, Roubicek T, Bartlova M, Mraz M, Lacinova Z, Haluzikova D, Kavalkova P, Matoulek M, Kasalicky M \& Haluzik M. Increased serum concentrations of macrophage inhibitory cytokine- 1 in patients with obesity and type 2 diabetes mellitus: the influence of very low calorie diet. European Journal of Endocrinology 2009161 397-404. (doi:10.1530/EJE-09-0417)

10 Wollert KC \& Kempf T. Growth differentiation factor 15 in heart failure: an update. Current Heart Failure Reports 2012 In press.

11 Daniels LB, Clopton P, Laughlin GA, Maisel AS \& Barrett-Connor E. Growth-differentiation factor- 15 is a robust, independent predictor of 11-year mortality risk in community-dwelling older adults: the Rancho Bernardo Study. Circulation 2011123 2101-2110. (doi:10.1161/CIRCULATIONAHA.110.979740)

12 Rohatgi A, Patel P, Das SR, Ayers CR, Khera A, MartinezRumayor A, Berry JD, McGuire DK \& de Lemos JA. Association of growth differentiation factor-15 with coronary atherosclerosis and mortality in a young, multiethnic population: observations from the Dallas Heart Study. Clinical Chemistry 201258 172-182. (doi:10.1373/clinchem.2011.171926)

13 Wollert KC, Kempf T, Peter T, Olofsson S, James S, Johnston N, Lindahl B, Horn-Wichmann R, Brabant G, Simoons ML, Armstrong PW, Califf RM, Drexler H \& Wallentin L. Prognostic value of growth-differentiation factor-15 in patients with nonST-elevation acute coronary syndrome. Circulation $2007 \mathbf{1 1 5}$ 962-971. (doi:10.1161/CIRCULATIONAHA.106.650846)

14 Anand IS, Kempf T, Rector TS, Tapken H, Allhoff T, Jantzen F, Kuskowski M, Cohn JN, Drexler H \& Wollert KC. Serial measurement of growth-differentiation factor-15 in heart failure: relation to disease severity and prognosis in the Valsartan Heart Failure Trial. Circulation 2010122 1387-1395. (doi:10.1161/ CIRCULATIONAHA.109.928846)

15 Bonaca MP, Morrow DA, Braunwald E, Cannon CP, Jiang S, Breher S, Sabatine MS, Kempf T, Wallentin L \& Wollert KC. Growth differentiation factor- 15 and risk of recurrent events in patients stabilized after acute coronary syndrome: observations from 
PROVE IT-TIMI 22. Arteriosclerosis, Thrombosis, and Vascular Biology 201131 203-210. (doi:10.1161/ATVBAHA.110. 213512)

16 Widera C, Pencina MJ, Meisner A, Kempf T, Bethmann K, Marquardt I, Katus HA, Giannitsis E \& Wollert KC. Adjustment of the GRACE score by growth differentiation factor 15 enables a more accurate appreciation of risk in non-ST-elevation acute coronary syndrome. European Heart Journal 201233 1095-1104. (doi:10.1093/eurheartj/ehr444)

17 Vila G, Riedl M, Anderwald C, Resl M, Handisurya A, Clodi M, Prager G, Ludvik B, Krebs M \& Luger A. The relationship between insulin resistance and the cardiovascular biomarker growth differentiation factor- 15 in obese patients. Clinical Chemistry 201157 309-316. (doi:10.1373/clinchem.2010.153726)

18 Torgerson JS, Hauptman J, Boldrin MN \& Sjostrom L. XENical in the prevention of diabetes in obese subjects (XENDOS) study: a randomized study of orlistat as an adjunct to lifestyle changes for the prevention of type 2 diabetes in obese patients. Diabetes Care 200427 155-161. (doi:10.2337/diacare.27.1.155)

19 Levey AS, Stevens LA, Schmid CH, Zhang YL, Castro AF III, Feldman HI, Kusek JW, Eggers P, Van Lente F, Greene T \& Coresh J. A new equation to estimate glomerular filtration rate. Annals of Internal Medicine 2009150 604-612.

20 Alberti KG, Eckel RH, Grundy SM, Zimmet PZ, Cleeman JI, Donato KA, Fruchart JC, James WP, Loria CM \& Smith SC Jr. Harmonizing the metabolic syndrome: a joint interim statement of the International Diabetes Federation Task Force on Epidemiology and Prevention; National Heart, Lung, and Blood Institute; American Heart Association; World Heart Federation; International Atherosclerosis Society; and International Association for the Study of Obesity. Circulation 2009120 1640-1645. (doi:10.1161/CIRCULATIONAHA.109.192644)

21 D'Orazio P, Burnett RW, Fogh-Andersen N, Jacobs E, Kuwa K, Kulpmann WR, Larsson L, Lewenstam A, Maas AH, Mager G, Naskalski JW \& Okorodudu AO. Approved IFCC recommendation on reporting results for blood glucose (abbreviated). Clinical Chemistry 200551 1573-1576. (doi:10.1373/clinchem.2005. 051979)

22 Matthews DR, Hosker JP, Rudenski AS, Naylor BA, Treacher DF \& Turner RC. Homeostasis model assessment: insulin resistance and $\beta$-cell function from fasting plasma glucose and insulin concentrations in man. Diabetologia 198528 412-419. (doi:10.1007/ BF00280883)

23 Kempf T, Horn-Wichmann R, Brabant G, Peter T, Allhoff T, Klein G, Drexler H, Johnston N, Wallentin L \& Wollert KC. Circulating concentrations of growth-differentiation factor 15 in apparently healthy elderly individuals and patients with chronic heart failure as assessed by a new immunoradiometric sandwich assay. Clinical Chemistry 200753 284-291. (doi:10.1373/ clinchem.2006.076828)
24 Meyer C, Pimenta W, Woerle HJ, Van Haeften T, Szoke E, Mitrakou A \& Gerich J. Different mechanisms for impaired fasting glucose and impaired postprandial glucose tolerance in humans. Diabetes Care 200629 1909-1914. (doi:10.2337/dc06-0438)

25 DeFronzo RA \& Abdul-Ghani M. Assessment and treatment of cardiovascular risk in prediabetes: impaired glucose tolerance and impaired fasting glucose. American Journal of Cardiology $2011 \mathbf{1 0 8}$ 3B-24B. (doi:10.1016/j.amjcard.2011.03.013)

26 Lind L, Wallentin L, Kempf T, Tapken H, Quint A, Lindahl B, Olofsson S, Venge P, Larsson A, Hulthe J, Elmgren A \& Wollert KC. Growth-differentiation factor- 15 is an independent marker of cardiovascular dysfunction and disease in the elderly: results from the Prospective Investigation of the Vasculature in Uppsala Seniors (PIVUS) Study. European Heart Journal 200930 2346-2353. (doi:10.1093/eurheartj/ehp261)

27 Carstensen M, Herder C, Brunner EJ, Strassburger K, Tabak AG, Roden M \& Witte DR. Macrophage inhibitory cytokine-1 is increased in individuals before type 2 diabetes diagnosis but is not an independent predictor of type 2 diabetes: the Whitehall II study. European Journal of Endocrinology 2010162 913-917. (doi:10.1530/EJE-09-1066)

28 Ahima RS. Connecting obesity, aging and diabetes. Nature Medicine 200915 996-997. (doi:10.1038/nm0909-996)

29 Minamino T, Orimo M, Shimizu I, Kunieda T, Yokoyama M, Ito T, Nojima A, Nabetani A, Oike Y, Matsubara H, Ishikawa F \& Komuro I. A crucial role for adipose tissue p53 in the regulation of insulin resistance. Nature Medicine 200915 1082-1087. (doi:10.1038/nm.2014)

30 Eggers KM, Kempf T, Lind L, Sundstrom J, Wallentin L, Wollert KC \& Siegbahn A. Relations of growth-differentiation factor-15 to biomarkers reflecting vascular pathologies in a populationbased sample of elderly subjects. Scandinavian Journal of Clinical and Laboratory Investigation $2012 \mathbf{7 2}$ 45-51. (doi:10.3109/ $00365513.2011 .626072)$

31 Olefsky JM \& Glass CK. Macrophages, inflammation, and insulin resistance. Annual Review of Physiology 201072 219-246. (doi:10.1146/annurev-physiol-021909-135846)

32 Jansson PA. Endothelial dysfunction in insulin resistance and type 2 diabetes. Journal of Internal Medicine 2007262 173-183. (doi:10.1111/j.1365-2796.2007.01830.x)

33 Macia L, Tsai VW, Nguyen AD, Johnen H, Kuffner T, Shi YC, Lin S, Herzog H, Brown DA, Breit SN \& Sainsbury A. Macrophage inhibitory cytokine 1 (MIC-1/GDF15) decreases food intake, body weight and improves glucose tolerance in mice on normal \& obesogenic diets. PLoS ONE 20127 e34868. (doi:10.1371/ journal.pone.0034868)

Received 30 May 2012

Revised version received 30 July 2012

Accepted 23 August 2012 